\title{
Factores Preditivos de Metastização Ganglionar Retroperitoneal no Cancro do Endométrio
}

\author{
Factors Predictive of Retroperitoneal Lymph Node Metastasis in \\ Endometrial Cancer
}

Cecília URZAL ${ }^{1}$, Rita SOUSA ${ }^{2}$, Vítor BALTAR ${ }^{2}$, Paulo CORREIA², Eugénia CRUZ ${ }^{3}$, Daniel PEREIRA DA SILVA ${ }^{2}$ Acta Med Port 2014 Jan-Feb;27(1):82-87

\section{RESUMO}

Introdução: Tem sido sugerido que o estadiamento completo possa ser omitido com segurança em doentes com carcinoma do endométrio e baixo risco de metástases ganglionares. Os objectivos do nosso trabalho foram a exploração do significado prognóstico de factores patológicos para disseminação ganglionar pélvica e para-aórtica e a validação do algoritmo da Clínica Mayo, de forma a identificar as doentes em que a linfadenectomia possa ser dispensada.

Material e Métodos: Efectuámos um estudo retrospectivo incluindo 208 doentes, para a avaliação de variáveis tumorais patológicas e metástases ganglionares. A análise estatística foi realizada através dos testes qui-quadrado, exacto de Fisher e $t$ de Student.

Resultados: A invasão miometrial $>50 \%(p<0,001)$, a invasão do colo $(p=0,001)$, a invasão dos espaços linfovasculares $(p=0,003)$ e a citologia peritoneal positiva $(p=0,03)$ constituíram preditores significativos de disseminação ganglionar retroperitoneal. A metastização ganglionar pélvica foi preditiva de metastização ganglionar para-aórtica $(p<0,001)$.

Discussão: O algoritmo da Clínica Mayo identificou as doentes sem metástases nos gânglios pélvicos ou para-aórticos com um valor preditivo negativo de $98,4 \%$ (61/62). A combinação de invasão miometrial $\leq 50 \%$ e ausência de invasão cervical ou linfovascular apresentou um valor preditivo negativo de $98,8 \%$ (79/80).

Conclusão: Apesar de os critérios da Clínica Mayo predizerem uma probabilidade muito reduzida de metástases ganglionares retroperitoneais, a combinação de invasão miometrial $\leq 50 \%$ e ausência de invasão cervical ou linfovascular teria evitado a linfadenectomia num número superior de mulheres.

Palavras-chave: Espaço Retroperitoneal; Estadiamento de Neoplasia; Neoplasias do Endométrio; Metástases Ganglionares; Factores Preditivos; Linfadenectomia; Portugal.

\section{ABSTRACT}

Introduction: It has been suggested that a complete staging may be safely omitted in endometrial carcinoma patients at low risk for lymph node metastasis. The purposes of our study were to explore the prognostic significance of pathologic factors for pelvic and paraaortic nodal spread and to validate the Mayo algorithm in order to identify patients in whom lymphadenectomy may be avoided.

Material and Methods: We conducted a retrospective review including 208 patients, regarding the evaluation of pathologic variables and nodal metastases. Statistical analysis was performed using the chi-square test, the Fisher exact test and the Student's $t$-test.

Results: Myometrial invasion $>50 \%(p<0.001)$, cervical invasion $(p=0.001)$, lymphovascular space invasion $(p=0.003)$ and positive peritoneal cytology $(p=0.03)$ were significant predictors of retroperitoneal lymph node dissemination. Pelvic lymph node metastases were predictive of positive paraaortic lymph nodes $(p<0.001)$.

Discussion: The Mayo algorithm identified patients without pelvic or paraaortic nodal metastases with a $98.4 \%$ negative predictive value $(61 / 62)$. Myometral invasion $\leq 50 \%$ and absence of cervical and lymphovascular invasion presented a negative predictive value of $98.8 \%(79 / 80)$.

Conclusion: Although the Mayo criteria predict a very low likelihood of retroperitoneal nodal metastases, the combination of myometral invasion $\leq 50 \%$ and absence of cervical or lymphovascular invasion would have safely avoided lymphadenectomy in a larger number of women.

Keywords: Endometrial Neoplasms; Lymphatic Metastasis; Lymph Node Excision; Neoplasm Staging; Predictive Value of Tests; Retroperitoneal Space; Portugal.

\section{INTRODUÇÃO}

Representando a neoplasia ginecológica mais frequente nos países desenvolvidos, o carcinoma do endométrio possui, em Portugal, taxas anuais de incidência e mortalidade cifradas em 10,3 e 1,9 por 100000 mulheres, respectivamente. Com base na clínica, na histologia e no perfil molecular, descrevem-se dois tipos de carcinoma do endométrio. Responsável por $80 \%$ dos casos, o tipo I relaciona-se com condições que configuram um estado prolongado de hiperestrogenismo não contrabalançado (anovulação, nuliparidade, menopausa tardia, administração de tamoxi- feno, cirrose hepática), atinge mulheres mais jovens e obesas e corresponde a tumores do tipo endometrióide, bem diferenciados e com melhor prognóstico. O tipo II associa-se a um endométrio atrófico, raramente exibe expressão de receptores hormonais, corresponde a tipos histológicos não endometrióide (células claras, serosos, carcinossarcomas) e cursa com invasão miometrial profunda e metastização ganglionar frequente. Globalmente, o carcinoma do endométrio acomete sobretudo mulheres na sexta e sétima décadas de vida, tendo a sua incidência vindo a aumen-

\footnotetext{
1. Departamento de Ginecologia e Obstetrícia. Centro Hospitalar Tondela-Viseu. Viseu. Portugal.

2. Serviço de Ginecologia. Instituto Português de Oncologia. Coimbra. Portugal.

3. Serviço de Anatomia Patológica. Instituto Português de Oncologia. Coimbra. Portugal.

Recebido: 07 de Julho de 2013 - Aceite: 11 de Novembro de 2013 | Copyright @ Ordem dos Médicos 2014
} 
tar em face da longevidade e obesidade crescentes das populações. A precocidade das manifestações clínicas, ao permitir mais de $75 \%$ dos diagnósticos num estádio inicial, concorre para que exiba um prognóstico geralmente favorável, com taxas de sobrevivência global (SG) aos cinco anos de 80 a $85 \% .{ }^{1} \mathrm{O}$ sistema de estadiamento cirúrgico adoptado pela Federação Internacional de Ginecologia e Obstetrícia (FIGO) em 1988 veio permitir uma melhor categorização das doentes em grupos prognósticos, o que facilita a comparação de resultados interinstitucionais e, essencialmente, contribui para uma maior adequação do tratamento adjuvante ao risco de recorrência tumoral (Tabela 1$).^{2}$

Merecendo destaque, o protocolo 33 desenvolvido pelo Gynecologic Oncology Group objectivou a presença de doença extra-uterina em $22 \%$ dos casos de carcinoma do endométrio clinicamente limitado ao útero, incluindo o compromisso do território ganglionar pélvico em $9 \%$ e para-aórtico em 5\%. ${ }^{3}$ Em consonância, passou a preconizar-se a linfadenectomia sistemática após a realização do lavado peritoneal, da biópsia de qualquer lesão suspeita e da histerectomia total extrafascial com anexectomia bilateral. A extensão anatómica da dissecção e o número de gânglios necessários para uma avaliação adequada foram deixados à consideração do cirurgião, de acordo com a estimativa de alto ou baixo risco de invasão neoplásica, sem todavia definir tais categorias. Transcorridas duas décadas, as estratégias operatórias ainda não se encontram padronizadas, e a decisão sobre a conduta tem permanecido na dependência de opiniões individuais, do biótipo da doente, das competências do cirurgião ou da gestão dos tempos operatórios, denotando a controvérsia subjacente ao seu impacto.
Apesar de a realização da linfadenectomia regional ser considerada fundamental para um estadiamento completo do carcinoma do endométrio, os opositores à sua indicação sistemática têm sugerido a definição de subgrupos de doentes de baixo risco, baseados nas características tumorais, em que o procedimento possa ser omitido. Pretendemos identificar factores patológicos preditivos de metastização ganglionar pélvica e/ ou para-aórtica, com o objectivo de formular um algoritmo que permita equacionar a necessidade de linfadenectomia de uma forma individualizada, e compará-lo com os critérios utilizados pela Clínica Mayo (Tabela 2). ${ }^{4}$

\section{MATERIAL E MÉTODOS}

Seleccionámos a população de estudo através da análise retrospectiva dos processos clínicos das 284 doentes submetidas a cirurgia primária por carcinoma do endométrio entre 2002 e 2011. A decisão de efectuar a linfadenectomia foi tomada pelo cirurgião, em função dos achados patológicos pré- e intra-operatórios e da estimativa do risco associado às co-morbilidades individuais. Incluímos apenas as 208 doentes submetidas a histerectomia total, anexectomia bilateral e linfadenectomia pélvica e/ou para-aórtica durante a intervenção, excluindo assim os 76 casos em que a dissecção ganglionar não foi realizada. As variáveis testadas quanto à possível associação com a probabilidade de metástases ganglionares incluíram a idade das doentes, o tipo histológico, o grau de diferenciação e o diâmetro do tumor, a profundidade de invasão do miométrio e a presença ou ausência de células neoplásicas no lavado peritoneal, infiltração do colo (epitélio ou estroma), invasão linfo-vascular e disseminação para as estruturas anexiais.

Tabela 1 - Sistema de estadiamento cirúrgico do carcinoma do endométrio (FIGO, 2009)

\begin{tabular}{cl}
\hline Estádio & \\
\hline I IA & Tumor limitado ao corpo uterino \\
IB & Invasão da metade interna do miométrio \\
II & Invasão da metade externa do miométrio \\
III & Tumor confinado ao útero, com invasão do estroma cervical \\
IIIA & Tumor com extensão loco-regional \\
IIIB & Invasão da serosa uterina e / ou anexos \\
IIIC & Invasão da vagina e / ou paramétrios \\
IIIC1 & Metástases ganglionares pélvicas e / ou para-aórticas \\
IIIC2 & Gânglios pélvicos invadidos \\
IV & Gânglios para-aórticos invadidos, com ou sem invasão dos gânglios pélvicos \\
IVA & Invasão da bexiga e / ou recto e / ou metástases à distância \\
IVB & Invasão da mucosa da bexiga e / ou do recto \\
\end{tabular}

Tabela 2 - Critérios de risco de metastização ganglionar retroperitoneal no carcinoma do endométrio conforme a Clínica Mayo

\begin{tabular}{lll}
\hline Factores patológicos & Baixo risco & Alto risco \\
\hline Tipo histológico & endometrióide & não endometrióide \\
Grau histológico & $\mathrm{G} 1 / \mathrm{G} 2$ & $\mathrm{G} 3$ \\
Invasão miometrial & $\leq 50 \%$ & $>50 \%$ \\
Diâmetro tumoral & $\leq 2 \mathrm{~cm}$ & $>2 \mathrm{~cm}$ \\
\hline
\end{tabular}


Todos os factores relativos ao tumor foram avaliados em exame pós-operatório, efectuado por patologista oncológico. A idade foi analisada enquanto variável contínua. A categorização histológica foi estabelecida de acordo com a classificação da Organização Mundial de Saúde e a determinação do grau de diferenciação glandular cumpriu as recomendações da FIGO. Os tumores de grau histológico 1 ou 2 (G1/G2) foram classificados como de baixo grau. Definiu-se invasão miometrial profunda como ultrapassando a sua metade interna, ou $>50 \%$. Considerou-se linfadenectomia como a remoção de pelo menos um gânglio linfático. Definiu-se disseminação pélvica e/ ou para-aórtica como o compromisso neoplásico das estruturas ganglionares à data da cirurgia. O estádio tumoral foi determinado em conformidade com o sistema da FIGO de 2009. Constituíram-se três grupos de casos cuja relação com as variáveis foi investigada: gânglios pélvicos positivos, independentemente do estado ganglionar para-aórtico (GPP), gânglios retroperitoneais (pélvicos ou para-aórticos) positivos (GRP) e gânglios para-aórticos positivos, independentemente do estado ganglionar pélvico (GPAP). A análise estatística foi realizada através do recurso ao sistema de software SPSS Statistics 17.0. A associação entre pares de variáveis categóricas foi testada através da aplicação dos testes exacto de Fisher e do Qui-quadrado, com os resultados expressos em número absoluto e percentagem. Para a comparação da distribuição da idade, utilizámos o teste $t$ de Student. As associações com um valor inferior ao limiar de 0,05 foram consideradas estatisticamente significativas e expressas com uma aproximação milesimal.

Tabela 3 - Distribuição da população de 208 doentes por estádio, tipo histológico e grau, de acordo com o estudo patológico definitivo

\begin{tabular}{lrr}
\hline Factores patológicos & $\boldsymbol{N}$ & $\%$ \\
\hline Estádio FIGO & 112 & \\
IA & 69 & 53,8 \\
IB & 8 & 33,2 \\
II & 1 & 3,8 \\
IIIA & 12 & 0,5 \\
IIIC1 & 6 & 5,8 \\
IIIC2 & & 2,9 \\
& & \\
Tipo histológico & & \\
Endometrióide & 184 & \\
Seroso papilar & 13 & 88,5 \\
Mucinoso & 2 & 6,2 \\
Células claras & 4 & 1,0 \\
Células escamosas & 2 & 1,9 \\
Carcinossarcoma & 3 & 1,0 \\
& & \\
& & \\
Grau histológico & & \\
G1 & & \\
G2 & & \\
G3 & & 10,1 \\
& & \\
\hline
\end{tabular}

\section{RESULTADOS}

Incluímos 208 doentes no estudo, com $87,0 \%$ dos casos no estádio I; $3,8 \%$ no estádio II e 9,2\% no estádio III. A média ( \pm DP) da idade foi de $64,7 \pm 8,6$ anos (mín. 46, máx. 81 anos), sendo de $68,8 \pm 8,4$ anos no grupo GRP e de 63,6 \pm 8,5 anos entre as restantes doentes. A frequência populacional das características patológicas testadas e a percentagem de invasão ganglionar na sua presença encontram-se sumariadas na Tabela 3.

Duzentas e seis doentes $(99,0 \%)$ foram submetidas a linfadenectomia pélvica e em 118 casos $(56,7 \%)$ foi realizada a dissecção ganglionar da área para-aórtica. Foi excisada uma média de 19,0 gânglios pélvicos (mín 4, máx 47) e de 4,5 gânglios para-aórticos (mín 1, máx 10) por doente, verificando-se que em $97,1 \%$ das linfadenectomias pélvicas foram removidos 10 ou mais gânglios e em $46,6 \%$ das linfadenectomias para-aórticas foi excisado um mínimo de cinco gânglios. Identificou-se disseminação tumoral nos gânglios pélvicos em 17 casos (8,3\% das linfadenectomias pélvicas) e a nível dos gânglios para-aórticos em seis $(5,1 \%$ das linfadenectomias para-aórticas), correspondendo a 18 doentes (cinco doentes apresentaram simultaneamente gânglios pélvicos e para-aórticos positivos). No grupo GPP, a invasão miometrial profunda $(p<0,001)$, a infiltração cervical $(p=0,003)$ e a invasão linfo-vascular $(p=0,002)$ foram associados com a disseminação ganglionar. No grupo GRP, os seguintes factores mostraram relação significativa com a invasão tumoral dos gânglios: invasão miometrial profunda $(p<0,001)$, infiltração cervical $(p=0,001)$, invasão linfo-vascular $(p=0,003)$, idade das doentes $(p=0,02)$ e citologia peritoneal positiva $(p=0,03)$ (Tabela 4$)$.

No grupo GPAP, a metastização ganglionar pélvica foi factor preditivo de invasão ganglionar para-aórtica $(p<$ 0,001). No estádio I, em tumores endometrióides G1/G2, a presença de invasão miometrial $>50 \%$ associou-se a uma incidência de $15,0 \%$ de metástases nos gânglios pélvicos e/ou para-aórticos. A presença de um tumor tipo endometrióide, G1/G2, com diâmetro $\leq 2 \mathrm{~cm}$ e invasão miometrial $\leq 50 \%$ teve um valor preditivo negativo para disseminação ganglionar retroperitoneal de 98,4\% (61/62). A verificação simultânea de invasão miometrial $\leq 50 \%$, ausência de infiltração do colo (epitélio ou estroma) e ausência de invasão linfo-vascular mostrou um valor preditivo negativo de $98,8 \%$ (79/80), tendo-se calculado uma incidência de metástases ganglionares em $15,3 \%$ dos casos que não cumpriram estes critérios (Tabela 5).

\section{DISCUSSÃO}

O valor diagnóstico da linfadenectomia encontra-se bem estabelecido, em oposição ao de outros métodos de avaliação pré e intra-operatória das cadeias ganglionares retroperitoneais. ${ }^{5}$ Acresce que o estado dos gânglios se assume como um importante factor de prognóstico no estádio I clínico, tendo-se registado uma probabilidade seis vezes superior de recorrência perante a confirmação da sua invasão (48 versus $8 \%$ ). ${ }^{6}$ A morbilidade que o procedimento acarreta tem constituído o principal argumento contra a 
Tabela 4 - Frequência das variáveis tumorais na população de 208 doentes e percentagem de invasão ganglionar pélvica e/ou para-aórtica na sua presença

\begin{tabular}{lccc}
\hline Factores patológicos & $\begin{array}{c}\text { Doentes } \\
\boldsymbol{n} / \%\end{array}$ & $\begin{array}{c}\text { Gânglios positivos } \\
\boldsymbol{n} / \%\end{array}$ & $\boldsymbol{p}$ \\
\hline Invasão miometrial $>50 \%$ & $89 / 42,8$ & $25 / 28,1$ & 0,001 \\
Infiltração cervical & $28 / 13,5$ & $12 / 42,9$ & 0,001 \\
Invasão linfovascular & $75 / 36,1$ & $25 / 33,3$ & 0,003 \\
Citologia peritoneal positiva & $38 / 18,3$ & $12 / 31,6$ & 0,030 \\
Diâmetro tumoral > 2 cm & $126 / 60,6$ & $30 / 23,8$ & 0,120 \\
Disseminação anexial & $2 / 1,0$ & $1 / 50,0$ & 0,280 \\
Tipo não endometrióide & $24 / 11,5$ & $7 / 29,2$ & 0,370 \\
G3 & $21 / 10,1$ & $4 / 19,0$ & 0,650 \\
\hline
\end{tabular}

Testes exacto de Fisher e do Qui-quadrado

Tabela 5 - Valor preditivo negativo dos algoritmos de decisão para dispensa de linfadenectomia em cirurgia de estadiamento por carcinoma do endométrio

Tipo endometrióide G1 / G2

Invasão miometrial $\leq 50 \%$

Diâmetro tumoral $\leq 2 \mathrm{~cm}$
Invasão miometrial $\leq 50 \%$

Ausência de invasão cervical

Ausência de invasão linfo-vascular sua prática sistemática, tendo em conta a idade avançada da maioria das doentes e a frequência de patologias associadas. ${ }^{7}$ A pesquisa do gânglio sentinela, constituindo uma alternativa promissora porque minimamente invasiva, encontra-se dificultada pela complexidade da drenagem linfática uterina. Diferentes técnicas de mapeamento linfático e locais de injecção têm sido ensaiados, estando a sua validação limitada pela heterogeneidade nas taxas de detecção e sensibilidade, pela falta de reprodutibilidade e pela necessidade de demonstrar uma clara vantagem custo-benefício.

Mas a questão em torno da qual se devem equacionar os riscos individuais é se a linfadenectomia comporta, realmente, um benefício diagnóstico (direccionando o tipo e a extensão da terapêutica adjuvante) ou terapêutico (influenciando directamente as taxas de sobrevivência global e livre de doença). Entre os ensaios prospectivos, e apesar de criticado pela falta de uniformidade nos critérios para dissecção ganglionar e radioterapia adjuvante e pelo reduzido número de gânglios excisados, o estudo ASTEC incluiu 1408 doentes de um total de 85 instituições e não mostrou diferença estatística na SG aos cinco anos entre os braços cirúrgicos com e sem linfadenectomia pélvica. ${ }^{8}$ Igualmente, o estudo PORTEC-2 negou o benefício da sua realização. Com um grupo de 514 doentes portadoras de carcinoma do endométrio em estádio I clínico, Panici et al limitaram a utilidade da linfadenectomia a um estadiamento mais correcto, sem repercussão sobre a SG aos cinco anos e com aumento significativo da morbilidade. O intervalo de tempo até ao diagnóstico de recorrência foi também seme-
Ihante entre os dois braços (14 versus 13 meses). ${ }^{9}$ Numa meta-análise que incluiu 1945 doentes randomizadas, com doença no estádio I clínico, não foi encontrada evidência de que a linfadenectomia diminua o risco de morte ou recorrência. ${ }^{10}$

Sem que o debate se tenha encerrado, e sob o desiderato de evitar o sub- ou o sobretratamento das doentes, tem vindo a tornar-se oportuna a consideração de factores preditivos do estado dos gânglios regionais. Assim, emerge a tendência para admitir a existência de um conjunto de doentes que dispensará um estadiamento cirúrgico completo, com base na estimativa de baixo risco de metastização ganglionar, e um grupo de alto risco que poderá beneficiar da sua realização para fins diagnósticos. A análise destes factores, clínicos e patológicos, tem constituído matéria de várias publicações. ${ }^{11,12}$ À semelhança dos nossos resultados, o grupo liderado por Tang identificou a invasão miometrial profunda, a infiltração cervical e a permeação linfática como os factores patológicos de risco de metastização ganglionar retroperitoneal em 310 doentes, atribuindo-lhes, respectivamente, odds ratio de 5,97; 2,72 e $12,01 .{ }^{13}$ A importância da invasão do espaço linfo-vascular também tem sido confirmada, com um valor preditivo negativo que alcança 95,6\% numa série de 628 doentes. ${ }^{14} \mathrm{Re}$ lativamente à invasão cervical, a sua presença havia sido associada a um risco de metastização pélvica de $15 \%$, cerca de três vezes inferior ao que calculámos ao considerar o envolvimento do estroma e/ou do epitélio. ${ }^{15}$ De forma interessante, a identificação de células neoplásicas no lavado peritoneal não representou um factor preditivo relevante no 
grupo GPP, mas a associação tornou-se estatisticamente significativa no grupo GRP. Com uma incidência de 10 a $17 \%$ nos casos de carcinoma do endométrio, a citologia peritoneal positiva é mais frequente perante a existência de doença extra-uterina. ${ }^{16}$ Sem que o significado da sua presença isolada enquanto factor independente esteja estabelecido de modo consensual, os dados apontam para que possa constituir um marcador negativo de prognóstico, reforçando outros indicadores pejorativos. ${ }^{17}$

A metastização nos gânglios pélvicos constituiu um factor preditivo de disseminação ganglionar para-aórtica, que se verificou em $29,4 \%$ dos casos de gânglios pélvicos positivos. As incidências referidas em publicações anteriores variam largamente entre 32 e $80 \%$, o que parece traduzir a influência do número de gânglios pélvicos invadidos e do padrão patológico de envolvimento nodal. ${ }^{18}$ Pelo contrário, o achado de metástases ganglionares para-aórticas sem compromisso neoplásico dos gânglios pélvicos é relativamente raro, cifrando-se na literatura entre 0 e $6 \% .{ }^{19} \mathrm{~A}$ disseminação para-aórtica isolada, cuja incidência no presente estudo foi de $0,05 \%$, poderá justificar-se pela existência de uma via de disseminação directa a partir do corpo uterino através dos ligamentos infundíbulo-pélvicos ou, em alternativa, pela presença de doença oculta a nível dos gânglios pélvicos, não identificada pelos métodos histológicos de rotina.

Salientando o significado do grau histológico e da profundidade de invasão do miométrio, Lee et al registaram $0,47 \%$ de metástases ganglionares entre 834 doentes com carcinoma de tipo endometrióide G1/G2 intra-epitelial, enquanto Cusidó et al verificaram invasão dos gânglios pélvicos e/ou para-aórticos em $37 \%$ dos casos com infiltração miometrial $>50 \%$ e em $45,5 \%$ dos tumores G3. ${ }^{20,21}$ No nosso trabalho, a invasão do miométrio $>50 \%$ foi o factor que mostrou a associação de maior magnitude com o risco de metastização ganglionar. Mesmo perante tumores do tipo endometrióide e com baixo grau de diferenciação, a presença de invasão miometrial profunda determinou um risco de $15 \%$ de envolvimento ganglionar pélvico e/ ou para-aórtico, superior ao risco de $9 \%$ calculado por Kwon et al no mesmo contexto. ${ }^{22}$ Numa análise multivariável prévia, quando avaliada paralelamente à distância do tumor à serosa e à percentagem de miométrio invadido, a profundidade de invasão miometrial mostrou ser o único factor de risco independente. ${ }^{23}$ Ainda que sem atingir relevo estatístico, verificámos uma tendência para a associação entre a incidência de invasão ganglionar retroperitoneal e o diâmetro tumoral $>2 \mathrm{~cm}$. O valor preditivo desta variável foi apontado por Schink et al, ao estudar um conjunto de 142 mulheres com carcinoma do endométrio no estádio I clínico, com detecção de metástases ganglionares em 4\% dos casos com tumores $\leq 2 \mathrm{~cm}$ e em $15 \%$ daqueles com tumores $>2 \mathrm{~cm} .{ }^{24} \mathrm{O}$ grupo de Mariani mostrou a ausência de disseminação em peça de linfadenectomia nos casos de carcinoma endometrióide, G1/G2, com invasão miometrial $\leq 50 \%$ e medindo $\leq 2 \mathrm{~cm}$, por oposição ao registo de invasão ganglionar em $22 \%$ das restantes doentes. ${ }^{4,25}$ Este algoritmo adoptado pela Clínica Mayo foi, mais recentemente, aplicado por outros autores que usaram o método de Kaplan-Meier, com uma incidência de metástases ganglionares de apenas $1,8 \%$ entre os 110 casos que satisfaziam as condições. ${ }^{26}$ Entre nós, a combinação destes factores identificou as doentes com carcinoma do endométrio no estádio I em que a linfadenectomia poderia ter sido omitida com um valor preditivo negativo de $98,4 \%$. Ao integrar, como parâmetros de selecção, os factores que no nosso estudo se associaram a um baixo risco de metastização ganglionar (invasão miometrial $\leq 50 \%$, ausência de invasão cervical e linfo-vascular), obtivemos um valor preditivo negativo ligeiramente superior $(98,8 \%)$. Realçamos que, com estes critérios, a linfadenectomia retroperitoneal teria sido dispensada com segurança em 79 doentes (38,0\%), o que se compara favoravelmente com a aplicação do algoritmo da Clínica Mayo, que evitaria o sobretratamento de 61 casos da nossa população (29,3\%). Reconhecem-se as limitações referentes à heterogeneidade da nossa amostra e ao número de casos com gânglios positivos incluídos no estudo, o que inviabiliza a aplicação de uma análise de regressão. Também, a dificuldade inerente à eventual avaliação da invasão linfo-vascular em exame patológico per-operatório. Finalmente, o escasso número médio de gânglios para-aórticos excisados impõe a confirmação da reprodutibilidade dos resultados a que respeitam. Sublinha-se o interesse de proceder a validações locais de um algoritmo adoptado em consenso nacional, mas originalmente desenvolvido sobre uma população da Região Centro-Oeste dos Estados Unidos da América, em que se regista uma percentagem escassa de descendência Portuguesa.

\section{CONCLUSÃO}

Revalidamos que a linfadenectomia pélvica e para-aórtica possa ser omitida nos casos de carcinoma do endométrio no estádio I, tipo endometrióide, G1/G2, com diâmetro tumoral $\leq 2 \mathrm{~cm}$ e invasão miometrial $\leq 50 \%$. Porém, o valor preditivo negativo para metastização ganglionar retroperitoneal da associação de invasão miometrial $\leq 50 \%$ e ausência de invasão cervical ou linfo-vascular foi ligeiramente superior na nossa população, com a vantagem de evitar um estadiamento cirúrgico completo num maior número de casos.

\section{CONFLITOS DE INTERESSE}

Os autores declaram a inexistência de conflitos de interesse na realização do presente trabalho.

\section{FONTES DE FINANCIAMENTO}

Não existiram fontes externas de financiamento para a realização deste artigo. 


\section{REFERÊNCIAS}

1. Creasman WT, Odicino F, Maisonneuve P, Quinn MA, Beller U, Benedet $\mathrm{JL}$, et al. Carcinoma of the corpus uteri. FIGO 26th Annual Report on the Results of Treatment in Gynecological Cancer. Int J Gynecol Obstet. 2006;95:105-43.

2. Sorosky JI. Endometrial cancer. Obstet Gynecol. 2008;111:436-47.

3. Creasman WT, Morrow CP, Bundy BN, Homesley H, Graham JE, Heller PB. Surgical pathologic spread patterns of endometrial cancer. A gynecologic oncology group study. Cancer. 1987;60:2035-41.

4. Mariani A, Webb MJ, Keeney GL, Haddock MG, Calori G, Podratz KC Low-risk corpus cancer: is lymphadenectomy or radiotherapy necessary? Am J Obstet Gynecol. 2000;182:1506-19.

5. Aalders JG, Thomas G. Endometrial cancer - Revisiting the importance of pelvic and para aortic lymph nodes. Gynecol Oncol. 2007;104:222-31.

6. Lurain JR, Rice BL, Rademaker AW, Poggensee LE, Schink JC, Miller DS. Prognostic factors associated with recurrence in clinical stage I adenocarcinoma of the endometrium. Obstet Gynecol. 1991:78;63-9.

7. Tozzi R, Malur S, Koehler C, Schneider A. Analysis of morbidity in patients with endometrial cancer: is there a commitment to offer laparoscopy? Gynecol Oncol. 2005;97:4-9.

8. Kitchener H, Swart AM, Qian Q, Amos C, Parmar MK. Efficacy of systematic pelvic lymphadenectomy in endometrial cancer (MRC ASTEC trial): a randomized study. Lancet. 2009;373:125-36.

9. Panici PB, Basile S, Maneschi F, Lissoni A, Signorelli M, Scambia G, et al. Systematic pelvic lymphadenectomy vs no lymphadenectomy in early-stage endometrial carcinoma: randomized clinical trial. J Natl Cancer Inst. 2008;100:1707-16.

10. May K, Bryant A, Dickinson HO. Lymphadenectomy for the management of endometrial cancer. Cochrane Database Syst Rev. 2010;20:CD007585.

11. Fotopoulou C, Savvatis K, Kraetschell R, Schefold JC, Lichtenegger W, Sehouli J. Systematic pelvic and aortic lymphadenectomy in intermediate and high-risk endometrial cancer: lymph-node mapping and identification of predictive factors for lymph-node status. Eur J Obstet Gynecol Reprod Biol. 2010;149:199-203.

12. Kamura T, Yahata H, Shigematsu T, Ogawa S, Amada S, Kaku T, et al. Predicting pelvic lymph node metastasis in endometrial carcinoma. Gynecol Oncol. 1999;72:387-91.

13. Tang X, Tanemura K, Ye W, Ohmi K, Tsunematsu R, Yamada T, et al. Clinicopathological factors predicting retroperitoneal lymph node metastasis and survival in endometrial cancer. Jpn J Clin Oncol. 1998;28:6738.

14. Guntupalli SR, Zighelboim I, Kizer NT, Zhang Q, Powell MA, Thaker PH, et al. Lymphovascular space invasion is an independent risk factor for nodal disease and poor outcomes in endometrioid endometrial cancer. Gynecol Oncol. 2012;124:31-5.

15. DiSaia PJ, Creasman WT, Boronow RC, Blessing JA. Risk factors and recurrent patterns in Stage I endometrial cancer. Am J Obstet Gynecol. 1985;151:1009-15.

16. Yalman D, Ozsaran Z, Anacak Y, Celik OK, Ozkök S, Ozsaran A, et al Postoperative radiotherapy in endometrial carcinoma: analysis of prognostic factors in 440 cases. Eur J Gynaecol Oncol. 2000;21:311-5.

17. Saga $Y$, Imai M, Jobo T, Kuramoto H, Takahashi K, Konno R, et al. Is peritoneal cytology a prognostic factor of endometrial cancer confined to the uterus. Gynecol Oncol. 2006;103:277-80.

18. Turan T, Hizli D, Sarici S, Boran N, Gundogdu B, Karadag B, et al. Is it possible to predict para-aortic lymph node metastasis in endometrial cancer? Eur J Obstet Gynecol Reprod Biol. 2011;158:274-9.

19. Mariani A, Keeney GL, Aletti G, Webb MJ, Haddock MG, Podratz KC Endometrial carcinoma: paraaortic dissemination. Gynecol Oncol. 2004;92:833-8.

20. Lee KB, Ki KD, Lee JM. The risk of lymph node metastasis based on myometrial invasion and tumor grade in endometrioid uterine cancers: a multicenter, retrospective Korean study. Ann Surg Oncol. 2009;16:28827.

21. Cusidó M, Fargas F, Rodríguez I, Alsina A, Baulies S, Tresserra F, et al. Role of lymphadenectomy in endometrioid endometrial cancer. Eur J Gynaecol Oncol. 2011;32:49-53.

22. Kwon JS, Mazgani M, Miller DM, Ehlen T, Heywood M, McAlpine JN, et al. The significance of surgical staging in intermediate-risk endometrial cancer. Gynecol Oncol. 2011;122:50-4

23. Kondalsamy-Chennakesavan S, Van Vugt S, Sanday K, Nicklin JL, Russell L, Lewis P, et al. Evaluation of tumor-free distance and depth of myometrial invasion as prognostic factors for lymph node metastases in endometrial cancer. Int J Gynecol Cancer. 2010;20:1217-21.

24. Schink JC, Lurain JR, Wallemark CB, Chmiel JS. Tumor size in endometrial cancer: a prognostic factor for lymph node metastasis. Obstet Gynecol. 1987;70:216-9.

25. Mariani A, Dowdy SC, Cliby WA, Gostout BS, Jones MB, Wilson TO, et al. Prospective assessment of lymphatic dissemination in endometrial cancer: a paradigm shift in surgical staging. Gynecol Oncol. 2008;109:11-8

26. Convery PA, Cantrell LA, Di Santo N, Broadwater G, Modesitt SC, Secord AA, et al. Retrospective review of an intraoperative algorithm to predict lymph node metastasis in low-grade endometrial adenocarcinoma. Gynecol Oncol. 2011;123:65-70. 


\title{
Factores Preditivos de Metastização Ganglionar Retroperitoneal no Cancro do Endométrio
}

\author{
Acta Med Port 2014:27:82-87
}

Publicado pela Acta Médica Portuguesa, a Revista Científica da Ordem dos Médicos

Av. Almirante Gago Coutinho, 151

1749-084 Lisboa, Portugal.

Tel: +351218428 215

E-mail: submissao@actamedicaportuguesa.com

www.actamedicaportuguesa.com

ISSN:0870-399X | e-ISSN: 1646-0758

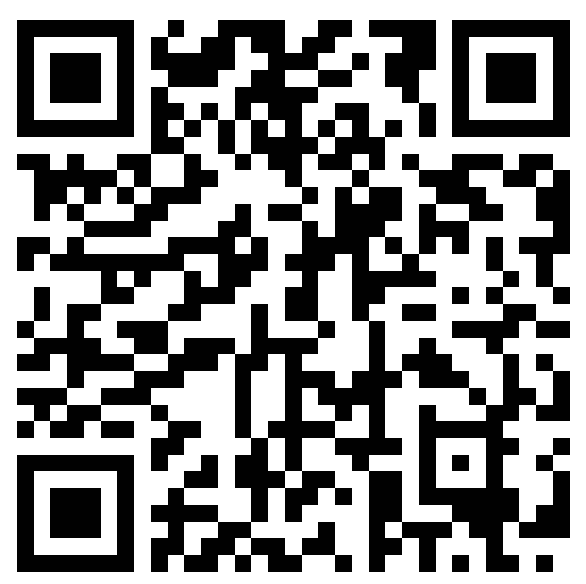

